IOS Press

\title{
Notice of retraction
}

IOS Press has retracted the following publication from its online content: [Cancer Biomarkers, 21(3) (2018), 661-673] $10.3233 / \mathrm{CBM}-170732$

Effects of exogenous IL-37 on the biological characteristics of human lung adenocarcinoma A549 cells and the chemotaxis of regulatory $T$ cells

Yu-Hua Chen ${ }^{\mathrm{a}}$, Bi-Yun Zhou ${ }^{\mathrm{b}}$, Guo-Cai Wu ${ }^{\mathrm{c}}$, De-Quan Liao ${ }^{\mathrm{a}}$, Jing Li ${ }^{\mathrm{a}}, \mathrm{Si}-\mathrm{Si}$ Liang ${ }^{\mathrm{d}}$, Xian-Jin Wu ${ }^{\mathrm{a}}, \mathrm{Jun}^{\mathrm{Fa}} \mathrm{Xu}{ }^{\mathrm{e}}$ Yong-Hua Chen ${ }^{\mathrm{f}}$, Xiao-Qing Dif ${ }^{\mathrm{f}}$ and Qiong-Yan $\operatorname{Lin}^{\mathrm{f}}$

${ }^{a}$ Department of Clinical Laboratory, Affiliated Hospital of Guangdong Medical University, Zhanjiang 524001, Guangdong, China

${ }^{\mathrm{b}}$ Department of Interventional Ward, Affiliated Hospital of Guangdong Medical University, Zhanjiang 524001 Guangdong, China

'Department of Hematology, Affiliated Hospital of Guangdong Medical University, Zhanjiang 524001, Guangdong, China

${ }^{\mathrm{d}}$ Department of Orthopedics, Affiliated Hospital of Guangdong Medical University, Zhanjiang 524001, Guangdong, China

e Department of Clinical Immunology, Institute of Laboratory Medicine Guangdong Medical University, Dongguan 523808, Guangdong, China

${ }^{\mathrm{f}}$ Department of Pathology, Affiliated Hospital of Guangdong Medical University, Zhanjiang 524001, Guangdong, China

The corresponding author contacted the editorial office requesting the article to be retracted. The authors claim that the experiments were not sufficiently standardized, which jeopardized the article results. During investigation, the editorial office found on PubPeer allegations of image manipulation, not refuted by the authors.

In this light the journal cannot condone publication of this paper and has decided to retract it from its online catalogue

$$
5
$$

